\title{
Evidence of Low Prevalence of Cystic Echinococcosis in the Catanzaro Province, Calabria Region, Italy
}

\begin{abstract}
Tommaso Manciulli, ${ }^{1,2,3 *}$ † Riccardo Serraino, ${ }^{4} \dagger$ Gian Luca D’Alessandro, ${ }^{3}$ Letizia Cattaneo, ${ }^{5}$ Mara Mariconti, ${ }^{1}$ Ambra Vola,${ }^{1}$ Francesco Taccari, ${ }^{6}$ Roberta Narra, ${ }^{3}$ Giuseppe De Vito, ${ }^{7}$ Enrico Maria Trecarichi, ${ }^{4}$ Maria Mazzitelli, ${ }^{4}$ Giovanni Matera, ${ }^{8}$ Adriano Casulli, ${ }^{9,10}$ Nadia Marascio, ${ }^{8}$ Enrico Brunetti, ${ }^{1,3}$ Francesca Tamarozzi, ${ }^{9}$ and Carlo Torti ${ }^{4}$

${ }^{1}$ Unit of Infectious and Tropical Diseases, IRCCS San Matteo Hospital Foundation, Pavia, Italy; ${ }^{2}$ PhD School of Experimental Medicine, University of Pavia, Pavia, Italy; ${ }^{3}$ Department of Clinical, Surgical, Diagnostic and Pediatric Sciences, University of Pavia, Pavia, Italy; ${ }^{4}$ Department of Medical and Surgical Sciences, Unit of Infectious and Tropical Diseases, "Magna Graecia" University, Catanzaro, Italy; ${ }^{5}$ Unit of Infectious Diseases, University of Naples-Federico II, Napoli, Italy; ${ }^{6}$ Institute of Clinical Infectious Disease, Catholic University of the Sacred Heart, Rome, Italy; ${ }^{7}$ Public Health Agency of the Catanzaro Province, Catanzaro, Italy; ${ }^{8}$ Department of Health Sciences, Unit of Clinical Microbiology, 'Magna Graecia' University, Catanzaro, Italy; ${ }^{9}$ Department of Infectious Diseases, WHO Collaborating Centre for Epidemiology, Detection and Control of Cystic and Alveolar Echinococcosis, Foodborne and Neglected Parasitic Diseases Unit, Istituto Superiore di Sanità, Rome, Italy; ${ }^{10}$ Department of Infectious Diseases, European Reference Laboratory for Parasites, Foodborne and Neglected Parasitic Diseases Unit, Istituto Superiore di
\end{abstract} Sanità, Rome, Italy

Abstract. Cystic echinococcosis (CE) is one of the neglected tropical diseases recognized by the WHO. Echinococcus granulosus sensu lato affects more than 1 million people worldwide and is responsible for high costs in the healthcare system. A clear knowledge of the prevalence of CE and its clinical characteristics could have an important impact on the approach to its diagnosis and to the public health planning of treatment and control interventions. We performed a prevalence study in four municipalities of Catanzaro Province, South Italy. This area is considered to be at high risk of $C E$ because of ovine breeding. We screened by abdominal ultrasound 2,426 volunteers, four of whom had abdominal CE. Given the need for prevention and control programs for CE in endemic areas of Italy, a detailed mapping of prevalence of CE, to complement data obtained through hospital discharge records, appears imperative.

\section{INTRODUCTION}

Cystic echinococcosis (CE) is a zoonotic infection caused by the larval stage of the cestode Echinococcus granulosus sensu lato. The parasite life cycle is maintained between canids, mainly dogs, which are definitive hosts harboring the adult stage of the parasite in the intestine, and livestock, mainly sheep, as intermediate hosts. ${ }^{1}$ Cystic echinococcosis has a cosmopolitan distribution and is particularly prevalent where sheep raising is practiced. ${ }^{2}$ Worldwide estimates suggest that 1.2 million individuals may be infected, with consistent economic losses for human treatment and animal production. ${ }^{3}$ The disease is included in the list of neglected tropical diseases for which the WHO advocates control. ${ }^{4}$ Furthermore, in high-income countries, CE may be considered an "orphan" disease as it is perceived as "rare" and not representing a real public health problem. ${ }^{5}$ Mediterranean areas of Europe have always been considered endemic for $\mathrm{CE},{ }^{6}$ and some studies have explored the clinical epidemiology of the disease using different methods. However, epidemiological data on CE are often lacking in both developing and highincome settings.

Studies based on hospital discharge records (HDR) have been performed in several countries. ${ }^{7-12}$ Other studies have analyzed clinical cohorts in single centers. ${ }^{13-20}$ However, hospital-based studies do not capture asymptomatic cases, and HDR do not account for outpatients. Differently, ultrasoundbased surveys describe more accurately the epidemiology of CE at population level. ${ }^{21,22}$ Such studies allow for the detection of CE active transmission to humans and can provide

\footnotetext{
*Address correspondence to Tommaso Manciulli, Department of Clinical, Surgical, Diagnostic and Pediatric Sciences, PhD School of Experimental Medicine, Viale Brambilla 54, Pavia 27100, Italy. E-mail: tommaso.manciulli01@universitadipavia.it

†These authors contributed equally to this work.
}

well-grounded estimates of disease prevalence. ${ }^{22}$ We present the results of an ultrasound population-based survey performed in four municipalities of Catanzaro Province in the Calabria region (Southern Italy), endemic for CE. ${ }^{9}$

\section{METHODS}

Study sites, subjects, and community outreach. Because of the unavailability of data on the prevalence of E. granulosus infection in animals, target sites were selected on the basis of the ratio between the number of sheep registered in the municipality and the human population, considering municipalities where the population ranged between 2,000 and 7,000 inhabitants. Indeed, the presence of CE in a geographical area has been correlated with the extent of sheep-raising activities, in particular with the density of sheep present in a territory; this has also been shown to correlate with the rates of CE-related hospitalizations. ${ }^{23,24}$ Therefore, we selected our target municipalities among those with the highest presence of previously diagnosed human CE cases as reported in the records of the Department of Infectious Diseases of "Mater Domini" Hospital of Catanzaro. The selected municipalities were Pianopoli (population = 2,613; territory of $24 \mathrm{~km}^{2}$ ), Tiriolo (population $=3,820$; territory $29 \mathrm{~km}^{2}$ ), Serrastretta (population $=3,082$; territory of $41 \mathrm{~km}^{2}$ ), and Guardavalle (population $=4,463$; territory of $60 \mathrm{~km}^{2}$ ). Figure 1 shows the localization of study sites.

Local communities were informed of the study using printed material (leaflets and posters) displayed in public locations and distributed in cooperation with public health and civil authorities and general practitioners based in the target sites. Public broadcasts through local television networks and press releases were also issued before the start of the screening activities at each site. The screening team, together with civil and health authorities, held a public meeting with communities before starting the work at each site to explain the purpose and 


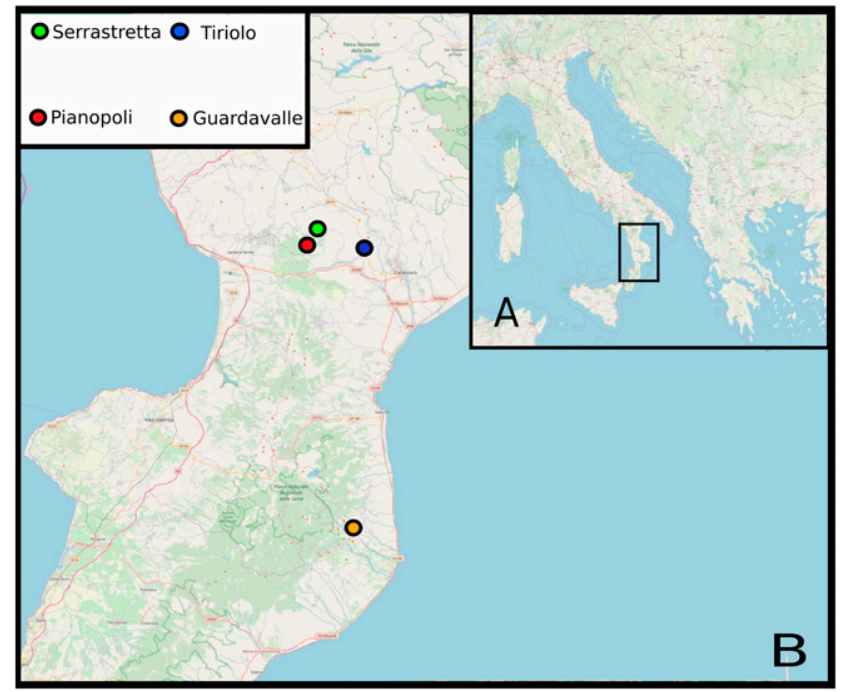

FIGURE 1. Sites in Calabria region (Italy) included in the study. (A) Map of Italy; the black box frames the province of Catanzaro where the study was conducted. (B) Magnification of the box framing the municipalities of Pianopoli, Tiriolo, Serrastretta, and Guardavalle where the study was carried out, with the localization of screening sites. This figure appears in color at www.ajtmh.org.

implementation of the study and to answer any questions arising from the audience.

Ultrasound screening. Public locations (e.g., town halls) provided by civil authorities were used as settings for the screenings. For practical reasons, convenience sampling was conducted on subjects voluntarily participating in the study, with the sole exclusion criteria of children younger than 5 years. Before enrollment, participants were given a brief overview of CE and information about the scope of the study, the execution of the abdominal ultrasound with the implications and limits of a screening activity, and the voluntary, freeof-charge nature of their participation.

Written informed consent was obtained from adult participants. In the case of minors or subjects who were not able to willingly provide consent, this was granted by their parents or legal guardians. Abdominal ultrasound examinations were conducted with four portable machines: Sonosite M-Turbo, Sonosite 180 plus (Fujifilm, Japan), Logiq V2 (General Electric, Boston, MA), and Z-6 (Mindray, China). Examinations were performed by physicians trained in general abdominal ultrasound and proficient in the diagnosis and staging of CE cysts according to the WHO Informal Working Group on Echinococcosis (IWGE) classification ${ }^{25}$. Images and video files of any lesion found during the examinations were recorded using an anonymized patient identification code and electronically stored. Participants were systematically asked if they had undergone any imaging examination in the 5 years before this screening and if they had received any previous diagnosis or treatment for CE.

Participants diagnosed with any medically relevant findings were provided with a written report and iconographic material, and referred for further clinical management to local national health centers liaising with the staff of the Department of Infectious Diseases of the University of Catanzaro. Individuals with CE were managed according to the WHO-IWGE Expert consensus for the clinical management of CE. ${ }^{25}$
Sample size. The minimum sample size of 1,522 subjects was calculated based on an estimated prevalence of $1 \%$ with a precision of $0.5 \%$ and $95 \%$ confidence level. For ethical reasons, participation to the screening was not denied to all subjects fulfilling the inclusion criteria who presented to the screening site, even after reaching an adequate sample size.

Ethical statement. The study was approved by the Ethics Committee of the Policlinico San Matteo Hospital Foundation, Pavia, Italy (prot. N. 20180060669). Data and images were pseudonymized and stored, and analyses were carried out in accordance with Italian legislation on personal data protection.

\section{RESULTS}

A total of 2,426 volunteers were screened in 28 days. Of these, $2,147(88.5 \%)$ were residents in the target municipalities, while $279(11.5 \%)$ came from villages within a few kilometers. Table 1 shows the number of the investigated population in screening sites. Among the total population screened, 1,506 (62.07\%) were females and the median age was 51 (range, 5-91) years; 1,471 (60.6\%) participants reported having undergone imaging examinations in the 5 years before the ultrasound performed as part of this study. CE cysts were found in four individuals $(0.16 \%)$; in all cases, they were single hepatic cysts in inactive stage (CE4). ${ }^{25}$ In all cases, the cysts were already known by the participant, and in two cases surgery had been proposed at diagnosis, notwithstanding the uncomplicated inactive stage. Five subjects (0.2\%) reported previous surgery for $\mathrm{CE}$, and no new abdominal CE cysts were detectable during the screening examination. Three participants $(0.1 \%)$, two of whom having a family history of $C E$, reported having a lesion suspect of $C E$, which was subsequently disproved, with a final diagnosis of unspecific liver calcifications in two cases, and multiple simple biliary and renal cysts in the other. Non-CE focal lesions found during the screening are detailed in Table 2.

\section{DISCUSSION}

The characteristics of CE make it difficult to clarify its epidemiology and burden in many regions of the world. ${ }^{26}$ Southern regions and islands of Italy are considered endemic for CE. ${ }^{9}$ However, although notification of all CE cases in Italy is mandatory, de facto only hospitalized cases with a primary or secondary diagnosis of CE are notified to the Italian Ministry of Health through HDR. Therefore, the only available data in Italy refer to the incidence of hospitalized CE cases, which represent only a fraction of the infected population. Moreover, HDR are burdened by inaccuracy and incompleteness. ${ }^{9}$

TABLE 1

Number of people screened and total resident population in the study sites in Catanzaro Province, Calabria region, Italy

\begin{tabular}{lccc}
\hline \multicolumn{1}{c}{ Study site } & Number screened & Total population & $\%$ \\
\hline Pianopoli & 544 & 2,613 & 20.8 \\
Serrastretta & 590 & 3,082 & 19.1 \\
Tiriolo & 457 & 3,820 & 11.9 \\
Guardavalle & 556 & 4,463 & 12.4 \\
Total $^{*}$ & 2,147 & 13,798 & 15.3 \\
\hline "Another 279 coming from municipalities adjacent to the target sites were also included, \\
bringing the total number of enrolled subjects to 2426.
\end{tabular}


TABLE 2

Non-Cystic echinococcosis focal lesions found during the screening in Catanzaro Province, Italy

\begin{tabular}{llrc}
\hline \multicolumn{1}{c}{ Diagnosis } & Organ & $N$ & $\%$ \\
\hline Cystic echinococcosis & Liver & 4 & 0.16 \\
Hemangioma & Liver & 61 & 2.51 \\
Simple cyst & Liver & 115 & 4.74 \\
Calcification & Liver & 36 & 1.48 \\
Focal liver lesion of suspected malignant & Liver & 5 & 0.2 \\
$\quad$ origin* & & & \\
Angiomyolipoma & Kidney & 21 & 0.86 \\
Simple cyst & Kidney & 295 & 12.1 \\
Suspect kidney cancer* & Kidney & 4 & 0.16 \\
Hemangioma & Spleen & 3 & 0.12 \\
Simple cyst & Spleen & 7 & 0.28 \\
Suspect simple cyst ${ }^{*}$ & Pancreas & 7 & 0,28 \\
Fibroma & Uterus & 63 & 2.59 \\
Suspect sarcoma* & Uterus & 3 & 0.12 \\
Simple cyst & Ovary & 62 & 2.55 \\
Suspect bladder cancer & Bladder & 1 & 0.04 \\
\hline${ }^{*}$ All patients with suspect lesions were referred for second-level & investigation as \\
appropriate (e.g., RMN of the abdomen for pancreatic cysts). & &
\end{tabular}

Based on HDR, the Calabria region shows an annual incidence of 2.6 cases $/ 100,000$ population. As such, it ranks as the fifth most endemic region in Italy. ${ }^{9}$ However, no precise animal data on CE infection are available, as no systematic surveillance at abattoirs, especially for small ruminants, is carried out in Italy. ${ }^{23}$ Recent work has used data modeling to estimate the prevalence of animal infection in Calabria, resulting in very low figures. ${ }^{23}$

In this epidemiological context, we decided to carry out an ultrasound-based survey in four villages in the Catanzaro Province of Calabria. Such studies can provide more accurate estimates of disease prevalence and information on the presence of ongoing transmission. ${ }^{21,22,27}$ Incidence of CE in the selected province, according to data derived from HDR, is 2-4 cases/100,000 population. ${ }^{9}$ In our study, the prevalence of abdominal CE by ultrasound was $0.16 \%$, far lower than what found in studies carried out in Morocco, Turkey, Bulgaria, and Romania, all areas where CE is considered as endemic as in Southern Italy. ${ }^{22,28}$ Also, we could not find cysts in active stages, which arguably indicates the lack of active transmission in the surveyed areas. The percentage of the resident population screened in the target municipalities (Table 1) allows us to confidently state that the four villages present a low prevalence of human CE. However, our screening included $0.7 \%$ of the whole population of the Catanzaro Province. Therefore, we cannot generalize our results to the whole province or region.

Interestingly, we did find cases misdiagnosed as CE and individuals with asymptomatic inactive CE cysts to whom unnecessary invasive treatment was proposed, contrary to what recommended by the WHO-IWGE. ${ }^{25}$ This highlights the well-known problem of poor knowledge of CE, its diagnosis, and clinical management outside referral centers, with attendant unnecessary risks for the patients and private and/or public health costs, especially when surgical management is applied indiscriminately. ${ }^{16,29-31}$ It is also interesting that $60 \%$ of volunteers presenting to our screening sites reported having already undergone a radiological examination able to detect CE in the 5 years before the screening. On the one hand, this may indicate a self-selection bias in our studied population, as it is possible that people more keen on undergoing medical checkups were also keener on taking advantage of the study screening. On the other hand, this also suggests that in settings where access to health care is available, such as in the case of the Italian universal coverage health system, a likely large proportion of the potentially infected population is and can be captured by the health system. In such context, a surveillance, based on the notification of all CE cases, is feasible, while population screening campaigns may have a less important role than in resource-poor settings. In line with this observation, a previous study looked at the rate of CE patients diagnosed in 19 ultrasound units in Italy, reporting a prevalence of CE of $0.7 \%$ over their studied cohort. ${ }^{32}$ In the last few years, the European Register of Cystic Echinococcosis (ERCE) has been set up. ${ }^{34}$ At the time of this publication, the total number of cases recorded in the ERCE from European centers was superior to the number of cystic and alveolar echinococcosis cases annually notified across Europe since the existence of the registry, and the total number of cases, including those entered by non-European centers, was more than 2,000 (P. Rossi, personal communication ${ }^{34}$ ). The ERCE, therefore, can be a valuable starting platform to implement a notification system of both inpatient and outpatient CE cases and a standardized case retrieval for observational clinical research.

To conclude, our survey suggests a low prevalence of human CE in the investigated areas, with likely absent or lowlevel ongoing transmission, in a region traditionally considered highly endemic for CE. The result of this survey, together with $60 \%$ of its participants declaring having undergone imaging examinations in the 5 years before this study and HDR data reporting CE cases in this area, suggests that the Italian universal coverage health system is capturing a large proportion of subjects with $\mathrm{CE}$, but these data fail to reach the official statistics. In this context, where virtuous health systems are in place, CE should be regarded as an orphan disease and patients should be managed by interdisciplinary teams in referral centers, thus avoiding the malpractice of surgery in inactive cysts. Surveillance activities are needed to better map, at both human and animal levels, the endemic areas for CE in Italy, to optimize public health policies on human and animal infection.

Received February 12, 2020. Accepted for publication May 8, 2020.

Published online September 21, 2020.

Acknowledgments: We thank Sam Goblirsch for editing the paper for grammar and style. We also thank all the civil authorities, the Public Health Department from ASP Catanzaro: Dr. Vincenzo De Giorgio and Rossella Scalise (deceased), the general practitioners, and the volunteers who took part in the study.

Financial support: This research was funded by ERANet L. A. C. (grant ELAC2015/T080544 to E. B. and A. C.) and the Italian Ministry of Health-NDTND project.

Authors' addresses: Tommaso Manciulli, Unit of Infectious and Tropical Diseases, IRCCS San Matteo Hospital Foundation, Pavia, Italy, and Department of Clinical, Surgical, Diagnostic and Pediatric Sciences, PhD School of Experimental Medicine, Pavia, Italy, E-mail: tommaso.manciulli01@ateneopv.it. Riccardo Serraino, Enrico Maria Trecarichi, Maria Mazzitelli and Carlo Torti, Department of Medical and Surgical Sciences, Unit of Infectious and Tropical Diseases, "Magna Graecia" University, Catanzaro, Italy, E-mails: riccardoserraino@ hotmail.it, em.trecarichi@unicz.it, m.mazzitelli88@gmail.com and torti@unicz.it. Gian Luca D'Alessandro, Roberta Narra, Department of Clinical, Surgical, Diagnostic and Pediatric Science, University of Pavia, Pavia, Italy, E-mails: gianluca.dalessandro01@ateneopv.it, robintells@hotmail.it. Letizia Cattaneo, Unit of Infectious Diseases, University of Naples-Federico II, Napoli, Italy, E-mail: letizia.cattaneo@gmail.com. Mara Mariconti and Ambra Vola, Unit of Infectious and Tropical Diseases, IRCCS San Matteo Hospital 
Foundation, Pavia, Italy, E-mails: maramariconti@libero.it and ambrvola@gmail.com. Francesco Taccari, Institute of Clinical Infectious Disease, Catholic University of the Sacred Heart, Rome, Italy, E-mail: taccari@hotmail.it. Giuseppe De Vito, Public Health Agency of the Catanzaro Province, Catanzaro, Italy, E-mail: giuseppe.devito@ asp.cz.it. Giovanni Matera and Nadia Marascio, Department of Health Sciences, Unit of Clinical Microbiology, "Magna Graecia” University, Catanzaro, Italy, E-mails: gm4106@gmail.com and nadiamarascio@ gmail.com. Adriano Casulli, Department of Infectious Diseases, WHO Collaborating Centre for Epidemiology, Detection and Control of Cystic and Alveolar Echinococcosis, Foodborne and Neglected Parasitic Diseases Unit, Istituto Superiore di Sanità, Rome, Italy, and Department of Infectious Diseases, European Reference Laboratory for Parasites, Foodborne and Neglected Parasitic Diseases Unit, Istituto Superiore di Sanità, Rome, Italy, E-mail: adriano.casulli@iss.it. Enrico Brunetti, Unit of Infectious and Tropical Diseases, IRCCS San Matteo Hospital Foundation, Pavia, Italy and Department of Clinical, Surgical, Diagnostic and Pediatric Science, University of Pavia, Pavia, Italy, E-mail: enrico.brunetti@unipv.it. Francesca Tamarozzi, Department of Infectious Diseases, WHO Collaborating Centre for Epidemiology, Detection and Control of Cystic and Alveolar Echinococcosis, Foodborne and Neglected Parasitic Diseases Unit, Istituto Superiore di Sanità, Rome, Italy, E-mail: f_tamarozzi@ yahoo.com.

\section{REFERENCES}

1. Deplazes $P$ et al., 2017. Global distribution of alveolar and cystic echinococcosis. Adv Parasitol 95: 315-493.

2. Romig T, Deplazes $P$, Jenkins D, Giraudoux P, Massolo A, Craig PS, Wassermann M, Takahashi K, de la Rue M, 2017. Ecology and life cycle patterns of echinococcus species. Adv Parasitol 95: 213-314.

3. Budke CM, Deplazes P, Torgerson PR, 2006. Global socioeconomic impact of cystic echinococcosis. Emerg Infect Dis 12: 296-303.

4. WHO, 2020. Neglected Tropical Diseases. Available at: https:// www.who.int/neglected_diseases/diseases/en/. Accessed February 2, 2020.

5. Junghanss T, da Silva AM, Horton J, Chiodini PL, Brunetti E, 2008. Clinical management of cystic echinococcosis: state of the art, problems, and perspectives. Am J Trop Med Hyg 79: 301-311.

6. Manciulli T, Mariconti M, Vola A, Lissandrin R, Brunetti E, 2017. Cystic echinococcosis in the Mediterranean. Curr Trop Med Rep 4: 235-244.

7. Lopez-Bernus A, Belhassen-García M, Alonso-Sardón M, CarpioPerez A, Velasco-Tirado V, Romero-Alegria Á, Muro A, Cordero-Sánchez M, Pardo-Lledias J, 2015. Surveillance of human echinococcosis in Castilla-Leon (Spain) between 2000-2012. PLoS Negl Trop Dis 9: e0004154.

8. Herrador Z, Siles-Lucas M, Aparicio P, Lopez-Velez R, Gherasim A, Garate T, Benito A, 2016. Cystic echinococcosis epidemiology in Spain based on hospitalization records, 1997-2012. PLoS Negl Trop Dis 10: e0004942.

9. Brundu D, PisedduT, Stegel G, Masu G, Ledda S, Masala G, 2014. Acta tropica retrospective study of human cystic echinococcosis in Italy based on the analysis of hospital discharge records between 2001 and 2012. Acta Trop 140: 91-96.

10. van Cauteren D, Millon L, de Valk H, Grenouillet F, 2016. Retrospective study of human cystic echinococcosis over the past decade in France, using a nationwide hospital medical information database. Parasitol Res 115: 4261-4265.

11. Carabin H, Balsera-Rodríguez FJ, Rebollar-Sáenz J, Benner CT, Benito A, Fernández-Crespo JC, Carmena D, 2014. Cystic echinococcosis in the province of Álava, north Spain: the monetary burden of a disease no longer under surveillance. PLoS Negl Trop Dis 8: e3069.

12. Mastrandrea S, Stegel G, Piseddu T, Ledda S, Masala G, 2012. A retrospective study on burden of human echinococcosis based on hospital discharge records from 2001 to 2009 in Sardinia, Italy. Acta Trop 123: 184-189.

13. Cappello E, Cacopardo B, Caltabiano E, Li Volsi S, Chiara R, Sapienza M, Nigro L, 2013. Epidemiology and clinical features of cystic hydatidosis in western Sicily: a ten-year review. World J Gastroenterol 19: 9351-9358.

14. Lissandrin R, Vola A, Tahiri S, Mariconti M, Manciulli T, Tamarozzi F, Brunetti E, 2018. Cystic echinococcosis in immigrants and Italians accessing a single referral center in Lombardy, Italy. Trav Med Infect Dis 32.

15. Petrone $L$ et al., 2013. Cystic echinococcosis in a single tertiary care center in Rome, Italy. Biomed Res Int 2013: 978146.

16. Escolà-Vergé L et al., 2019. Retrospective study of cystic echinococcosis in a recent cohort of a referral center for liver surgery. J Gastrointest Surg 23: 1148-1156.

17. Romero-Alegria $A$, Belhassen-García $M$, Alonso-Sardón $M$, Velasco-Tirado V, Lopez-Bernus A, Carpio-Pérez A, Bellido JLM, Muro A, Cordero M, Pardo-Lledias J, 2017. Imported cystic echinococcosis in western Spain: a retrospective study. Trans R Soc Trop Med Hyg 110: 664-669.

18. Zammarchi L, Vellere I, Stella L, Bartalesi F, Strohmeyer M, Bartoloni A, 2017. Spectrum and burden of neglected tropical diseases observed in an infectious and tropical diseases unit in Florence, Italy (2000-2015). Intern Emerg Med 12: 467-477.

19. Gironé G, Mateo C, Gaya V, Usó J, Mínguez C, Roca B, Ramos $\mathrm{JM}, 2015$. Admissions for imported and non-imported parasitic diseases at a general hospital in Spain: a retrospective analysis. Travel Med Infect Dis 13: 322-328.

20. David Morais JA, 2007. Human hydatidosis in the district of Evora, Portugal: a clinical-epidemiological study over a quarter of a century. Acta Med Port 20: 1-10.

21. Tamarozzi $F$ et al., 2018. Prevalence of abdominal cystic echinococcosis in rural Bulgaria, Romania, and Turkey: a cross-sectional, ultrasound-based, population study from the HERACLES project. Lancet Infect Dis 18: 769-778.

22. Chebli $\mathrm{H}$ et al., 2017. Human cystic echinococcosis in Morocco: ultrasound screening in the Mid Atlas through an ItalianMoroccan partnership. PLoS Negl Trop Dis 11: e0005384.

23. Loi F, Laddomada A, Coccollone A, Marrocu E, Piseddu T, Masala G, Bandino E, Cappai S, Rolesu S, 2019. Socio-economic factors as indicators for various animal diseases in Sardinia. PLoS One 14: e0217367.

24. Colombe S, Togami E, Gelaw F, Antillon M, Fuentes R, Weinberger DM. 2017. Trends and correlates of cystic echinococcosis in Chile: 2001-2012. PLoS Negl Trop Dis 11: 2001-2012.

25. Brunetti E, Kern P, Vuitton DA, 2010. Expert consensus for the diagnosis and treatment of cystic and alveolar echinococcosis in humans. Acta Trop 114: 1-16.

26. Brunetti E, Garcia HH, Junghanss T, 2011. Cystic echinococcosis: chronic, complex, and still neglected. PLoS NegI Trop Dis 5: 3-7.

27. MacPherson CN, Romig T, Zeyhle E, Rees PH, Were JB, 1987. Portable ultrasound scanner versus serology in screening for hydatid cysts in a nomadic population. Lancet 2: 259-261.

28. WHO, 2012. Echinococcosis - Epidemiology. Available at: https:// www.who.int/echinococcosis/epidemiology/en/. Accessed February 2, 2020.

29. Nabarro LE, Amin Z, Chiodini PL. 2015. Current management of cystic echinococcosis: a survey of specialist practice. Clin Infect Dis 60: 721-728.

30. Narra R, Maestri M, Budke CM, Tamarozzi F, Mariconti M, Nicoletti GJ, Rinaldi F, Brunetti E. 2016. Costs associated with surgically treated cases of abdominal cystic echinococcosis: a single center's experience from 2008 to 2014, Pavia, Italy. Am $J$ Trop Med Hyg 95: 405-409.

31. Vola A, Manciulli T, De Silvestri A, Lissandrin R, Mariconti M, SilesLucas M, Brunetti E, Tamarozzi F, 2019. Diagnostic performances of commercial ELISA, indirect hemagglutination, and western blot in differentiation of hepatic echinococcal and nonechinococcal lesions: a retrospective analysis of data from a single referral centre. Am J Trop Med Hyg 101: 1345-1349.

32. Caremani M, Maestrini R, Occhini U, Sassoli S, Accorsi A, Giorgio A, Filice C. 1993. Echographic epidemiology of cystic hydatid disease in Italy. Eur J Epidemiol 9: 401-404.

33. Rossi P, Tamarozzi F, Galati F, Pozio E, Akhan O, Cretu CM, Vutova K, Siles-Lucas M, Brunetti E, Casulli A, 2016. HERACLES extended network. The first meeting of the European Register of Cystic Echinococcosis (ERCE). Parasit Vectors 9: 243.

34. EFSA, 2019. The European Union One Health 2018 zoonoses report. EFSA J 17: 5926. 